\title{
Le dessableur cloisonné d'Innertkirchen
}

\section{The Innertkirchen partitioned sand trap}

\author{
PAR P. GARIEL \\ INGŔNIEUR A LA SOCIÉté GRENOBLOTSE D'ÉTUdES ET D'APPLICATIONS HYDRAULIQUES \\ (SOGREAH, GRENOBLE)
}

\begin{abstract}
C'est sur une adduction secondaire de la centrale d'Innertkirchen (Suisse) qu'a été installé le premier dessableur cloisonné important. L'auteur décrit l'ouvrage classique primitivement adopté et sa transformation par l'adjonction d'un cloisonnement muni d'arganes de recueil des dépóts. L'efficacité se trouve ainsi grandement augmentée du fait de la réduction de la hauteur de chate libre des grains. Gràce aux dispositions adoptées à la suite d'une élude sur modèle réduit, l'ouvrage arrête environ les trois quarts des grains de un dixième de millimèlre et les dépôts sont évacués sans aucune difficulté moyennant une perte d'ean très iaible.
\end{abstract}

\begin{abstract}
The first important partitioned sand trap was installed in an auxilliary supply tunnel of the Innertlitchen power station, Switzerland. The author describes the conventional structure as originally taken together with the alterations carried out by the attachment of a partition assembly fitted with deposit receivers. In this way the efficiency is greatly improved owing to the reduction in free fall height of the particles. As a result of the modifications made after a scale moael investigation, the structure stops about three quarters of the approximately $0.1 \mathrm{~mm}$ particles and the deposits are taken anay withont any difficulty and with only a bery slight water loss.
\end{abstract}

\section{INTRODUCTION}

A la fin du printemps de 1955 , une nouvelle adduction d'eau à la centrale d'Innertkirchen ćtait mise en service. Elle avait pour but d'alimenter cette centrale avec les eaux de fonte des glaciers de la Trift et de Stein et celles du vallon de Gadmen, épargnant ainsi des eaux lace lacs artificiels précieuses pour l'hiver.

Cette nouvelle adduction aboutissant dans la galerie d'amenée posait un problème de dessablage important : n'était-il pas dangereux d'envoyer des eaux glaciaires, donc forcément sableuses, sur des lurbines qui ne s'usaient pas auparavant puisqu'elles étaient alimentées par des eaux de lacs?

Pour réduire ce risque sérieux au minimum, on avait prévu un dessableur classique à chacune des trois nouvelles prises d'eau, mais en 1953, les ouvrages élant déjà en construction, une manouvre d'ailleurs volontaire des vannes, ayant pour but de curer un des lacs supérieurs des dépôts qu'il contenait, eut pour résultat d'user les machines en onze jours infiniment plus que pendant les onze années d'exploitation noŕmale qui venaient de s'écouler. Pourtant les grains responsables de l'usure étaient surtout du limon inférieur à 50 microns avec un grain moyen assez voisin de 5 innicrons.

Cette expérience incita le Maître de l'Guvre à réexaminer les précautions de dessablage qui avaient été prises en vue de les augmenter si la chose était possible. Cela apparut comme superflu pour deux des nouvelles prises, mais pour la troisième, celle du glacier de Trift, il parut indispensable de faire un effort spécial vu l'abondance des sables contenus dans les eaux à certaines époques.

Il ne s'agissait pas, hien entendu, d'arrêter les grains de limon (indécantables sinon dans des lacs) qui usent les machines à coup sûr mais lentement, mais les sables plus grossiers qui peuvent provoquer des usures massives et soudaines lor's des violentes intempéries.

La Société des Kraftwerke Oberhasli décida 
done de transformer le dessableur de la Trift en dessableur cloisonné, suivant les dispositions brevetées par les Etablissements Neyrpic, qui permetlait une amélioration considérable tout en restant économiquement acceptable.

L'ouvrage étant déjà à moitié construit, il fallait apporter cette amélioration dans un cadre existant, ce qui entraînait des sujétions supplé- mentaires. De plus, en raison du caractère de nouveauté des améliorations proposées, il fut décidé d'effectuer une étude sur modèle réduit de l'ensemble de l'ouvrage que nous allons situer dans son cadre et décrirc rapidement.

Comme on le verra en terminant, on est parvenu à un ouvrage d'exploitation facile et d'une haute efficacité.

\section{I. - L'OUVRAGE ET SON CADRE}

Le torrent de la Trift, qui descend du glacier du mème nom en quelque sorte adossé au glacier du Rhône, est coupé à la cote 1324,50 par un petit barrage poids qui forme la retenue (fig. 1). La configuration des lieux, la nature du terrain, les nécessités imposées par le passage à proximité de la galerie provenant des prises plus en amont, ont conduit à un ensemble original qui fut conçu par M. Juillard, ingéniem-conseil à Berne, à cette époque ingénieur en chef des Kraftwerke Oberhasli.

Le vallon est étroit, ses flancs couverts d'éboulis instables granitiques. Le barrage, d'une longueur de $78 \mathrm{~m}$ en crête, est en deux parties : la partie déversante, pavée de granit, est creuse, pour des raisons d'économie de béton et de circulation du personnel pour accéder à la chambre qui contient la vanne de fond, du type secteur.

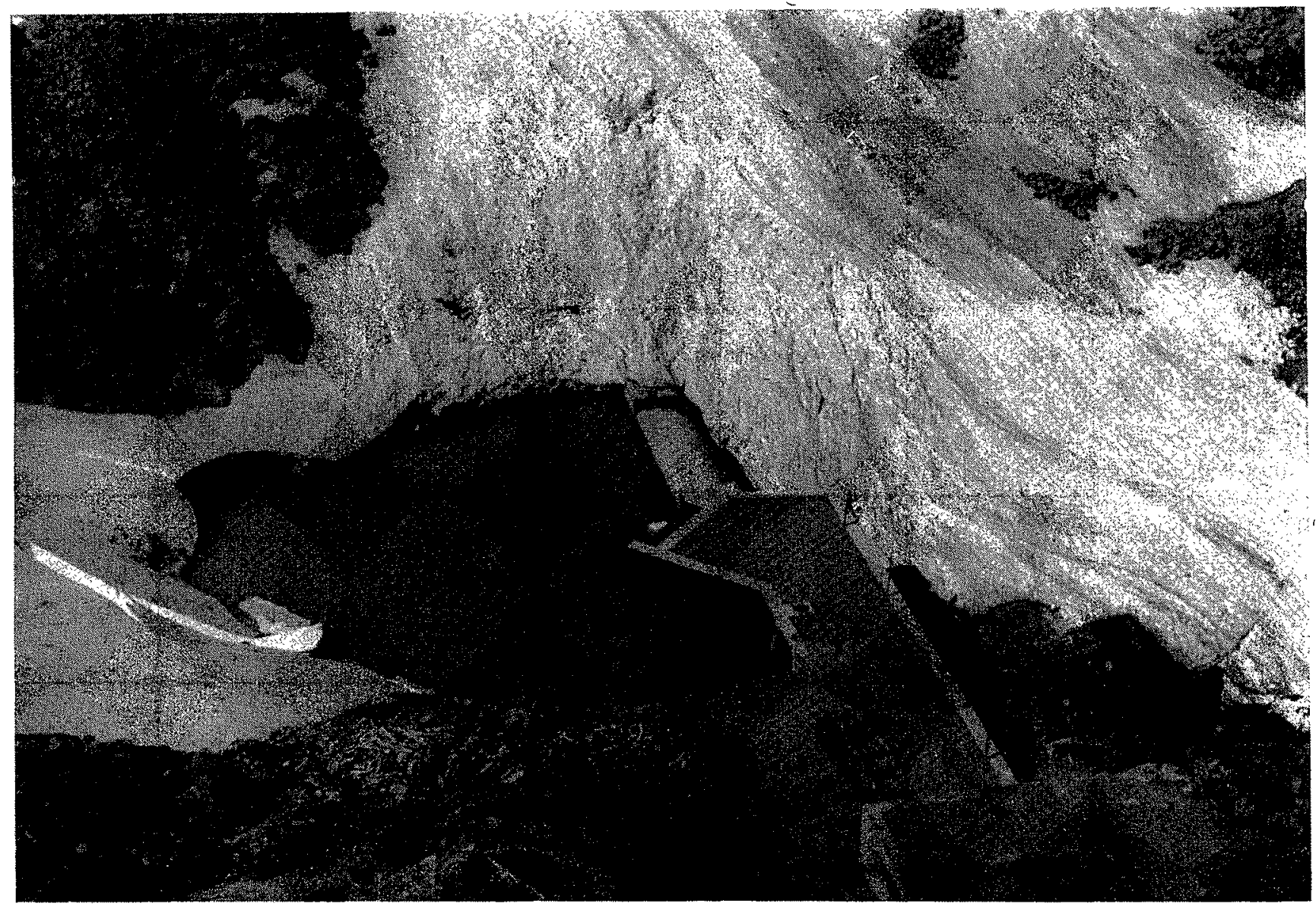

Fig. 1.

Vue d'ensemble du barrage déversant contenant le dessableut. 
L'aulre partie, còté rive droite, plus élevée, est creuse également, car elle contient la grande chambre de dessablage dont if va être question et un long couloir de circulation. Le tout est couvert d'une forte dalle surmontée d'une épaisse couche de cailloutis protectrice contre les chutes de rochers el les avalanches, dont la photographie montre des résidus.

Dans cetle zone, la pente du torrent est de $18,5 \%$. Le débit de prise est au maximum de $6,75 \mathrm{~m} 3 \mathrm{~s}$, soit environ le quart du débit absorbé par la centrale, mais, les jours de grandes crues, le torrent débite bien davantage; les eaux sont alors jaunâtres, chargées de limon glaciaire et de sable siliceux.

Le glacier occupe $90 \%$ du bassin versant qui est entièrement composé de roches cristallines: granites ou schistes métamorphiques. Les sables en suspension sont presque exclusivement quartzeux, à grains possédant des arêtes très tranchantes.

On voit sur la photographie la prise, avec ses grilles fines, arrangée pour prendre spécialement l'eau de surface de la retenue. Sa disposition oblige l'eau à franchir un coude à $45^{\circ}$ pour pénétrer dans la chambre de dessablage. On peut remarquer que la surface en plan de cette chambre ne represente qu'une fable fraction de la bien avec les formes du rocher et les nécessités de l'exploitation.

Selon le projet initial, l'eau purifiée devait s'évacuer par deux fentes ménagces dans le plafond et par un orifice aval alimentant un collecteur latéral aboutissant à un puits dont la section était calibrée pour débiter $6,75 \mathrm{~m}^{3} / \mathrm{s}$ pour un niveau amont à la cote de $1.324,50$.

Une petite galerie munie d'une vanne à glissement aboulissait au fond de l'ouvrage, destinie à évacuer les dépôts pall des vidanges complètes de durce appropriée.

Comme on le voit sur celle mème figure, e'esl par l'installation d'un cloisonnement appropric dans la partie aval que l'ouvrage a élé amélioré.

Vu la forme de la chambre, il n'est pas très facile de calculer exactement l'effiencité de ce dessableur avant modification : la forte divergence à l'entrée, le coude à $45^{\prime \prime}$, la profondeur variable, l'extraction de l'eau en trois points du plafond compliquent le caleul si on le reut exact.

On peut simplifier en considérant simplement la partie rectiligne de la chambre, de $6 \mathrm{~m}$ de largeur et $30 \mathrm{~m}$ de longueur, soit une surface en plan de $180 \mathrm{~m}^{2}$. On peut montrer qu'en eau calme, la vitesse de chute du grain le plus fin se trouvant à la partie supérieure de la section

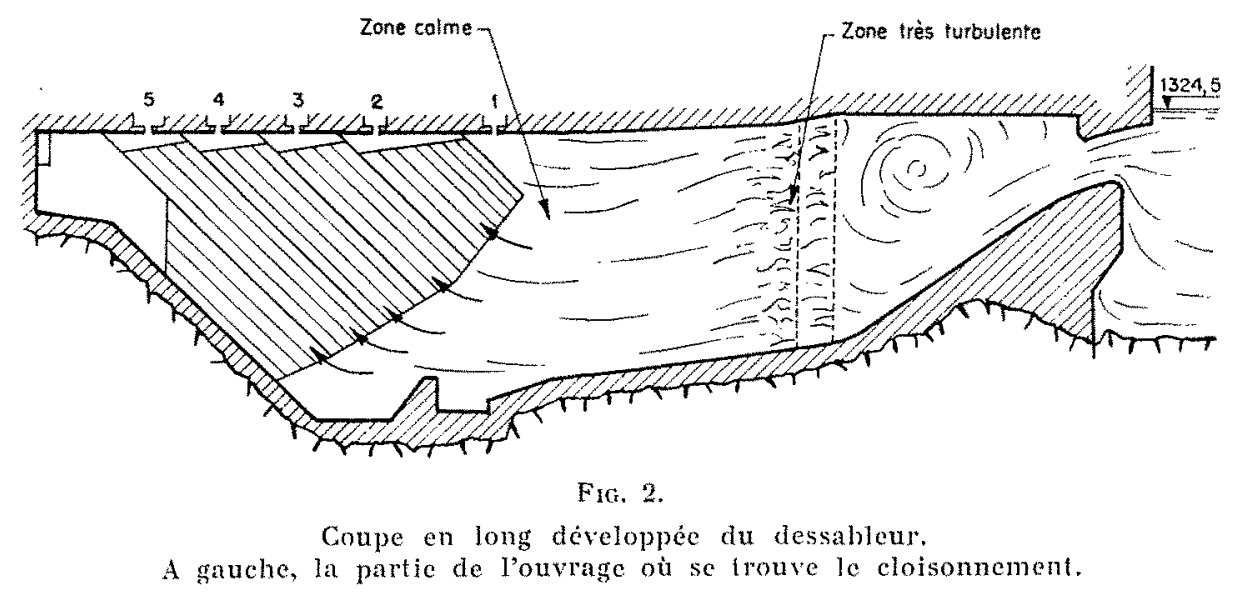

surface de la retenue, ce qui est une disposition excellente facilitant la décantation de beaucoup de sable avant que l'eau ne pénitre dans le dessableur.

La forme de la chambre de dessablage apparait sur la figure 2 qui est une coupe en long, développée à cause du coude à $45^{\circ}$ entre les perties amont et aval de louvrage.

La largeur est de $6 \mathrm{~m}$ sur toute Ia longueur de la chambre qui est de $50 \mathrm{~m}$ au total, dont $34 \mathrm{~m}$ de plafond pour la partie rectiligne aval.

Le radier est incliné à $75 \%$ dans la partie amont, horizontal dans la zone de profondeur maximum $(13 \mathrm{~m}), 10 \%$ au centre et remonte à $45^{\circ}$ à l'extrémité aval. Cette forme s'harmonisait d'entrée qui est tolalement décanté, a pour valeur :

$$
W=\frac{Q}{S}=\frac{6,75 \mathrm{~m}^{2} / \mathrm{s}}{80 \mathrm{~m}^{2}}=37 \mathrm{~mm} / \mathrm{s} .
$$

ce qui correspond à un grän de silice sphérique de $3 / 10^{\circ *}$ de $\mathrm{mm}$ environ tombant dans l'eau à $10^{\prime \prime}$.

Ce calcul est optimiste car il nóglige lous les phénomènes parasites tels que la turbulence et les perturbalions venant de l'entrée. Si l'on admel, avee ce calcul, que l'on décante $100 \%$ des grains de $3 / 10^{\text {ms }}$ et que les grains plus fins sont décantés proportionnellement au rapport de leur vitesse de chule à celle du grain de $3 / 10^{*}$, les 
efficacité sur ces diflérents grains seraient celles du tableau I:

TABLEAU I

\begin{tabular}{|lr|c|c|c|c|c|c|c|c|}
\hline Diamètre & en mm & 0,30 & 0,25 & 0,20 & 0,15 & 0,10 & 0,075 & 0,050 \\
\hline Efficacité & $\%$ & 100 & 75 & 57 & 36 & 18 & 8 & 3.5 \\
\hline
\end{tabular}

Ces valeurs sont plutôt exagérées. Cependant, ce dessableur eùt été aussi efficace que les ouvrages courants; d'ailleurs, on n'aurait pas trouvé à l'aval des grains de l'ordre de $3 / 10^{\prime \prime s}$, pour la raison qu'ils sont presque tous arrêtés par la retenue elle-même.

\section{II. - CLOISONNEMENT ET MODELLE REDUIT}

L'augmentation importante de l'efficacité de l'ouvrage a été obtenue par l'installation dans la partie aval d'un cloisonnement muni d'organes de recueil appropriés.

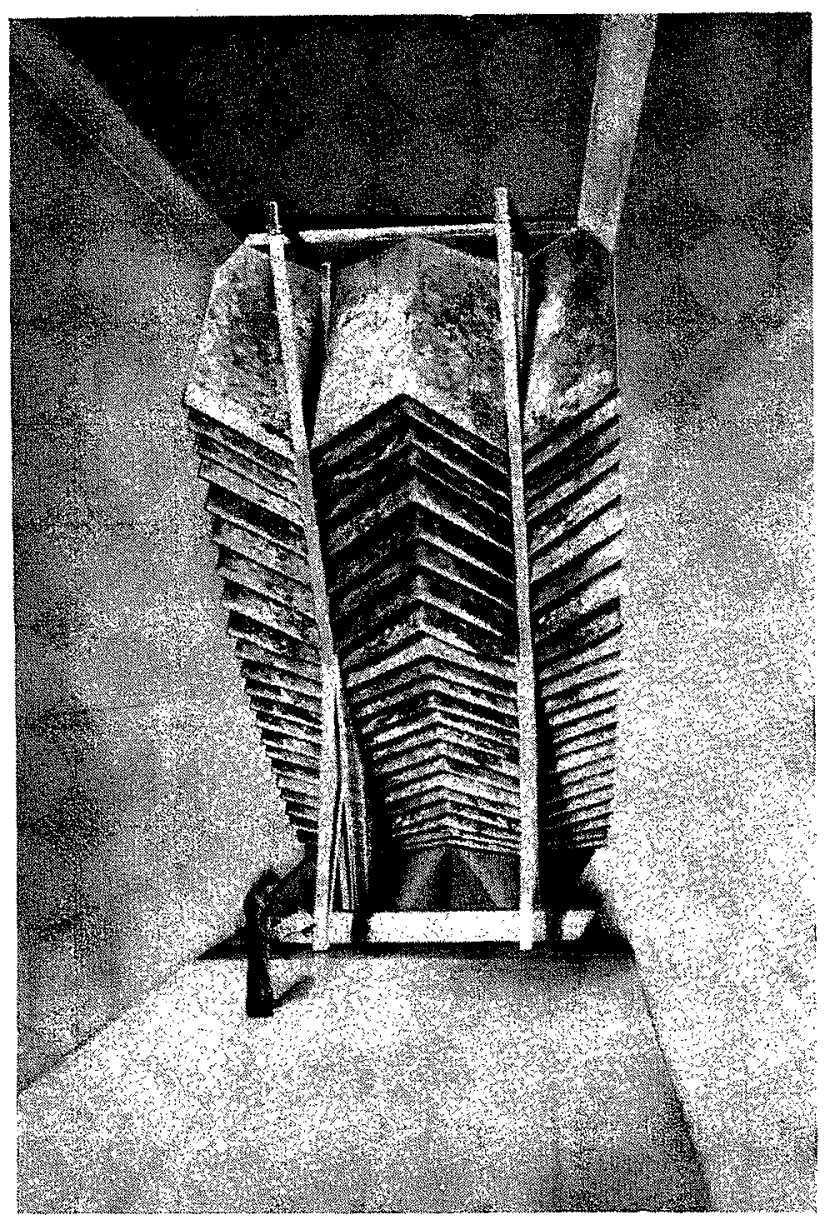

Fid. 3.

Vue du cloisonnement de la chambre de dessablage (d'après modète réduit).

Comme nous avons déjà eu l'occasion de l'exposer (1), et la chose est bien connue, le fait

(1) La Honille Blanche, $\mathrm{N}^{\circ}$ special $\mathrm{B}$, oetobre 1951, P. Ganié: «Nouvelles dispositions de dessableur», p. 630 à 639 . de disposer dans un grand volume des cloisons multiples augmente l'efficacité de la décantation puisque la hauteur de chute libre des grains est divisée dans le rapport de la profondeur totale à la distance verticale séparant deux cloisons. On a done intérêt à les rapprocher le plus possible. II faut, de plus, que les cloisons soienl assez inclinées pour que les dépôts puissent y glisser librement, mais c'est insuffisant. Il est essentiel que des organes de recueil de forme appropriée collectent les dépôts quî̀ s'écoulent des cloisons et les conduisent vers la partie basse de l'ouvrage sans remise en suspension notable, autrement l'efficacité du système se trouverait fortement compromise, sinon ruinée.

Ces organes de recueil conduisent les dépòts jusqu'à la fosse de stockage, au fond de laquelle se trouvent les points d'évacuation.

Pour des raisons d'hydraulique et de construction, on a été amené à réaliser la disposition représentée sur la figure 3 qui comporte une travée centrale et deux demi-travées latérales : les plaques sont en forme de toit an milieu et de demi-toit latéralement. Elles s'appuient sur deux charpentes qui constituent des cloisons creuses verticales qui servent à la fois de supports et d'organes de recueil. L'ensemble a été construit en alliage léger aluminium-magnésium et pesait environ 25 tonnes.

L'ouvrage se prêtait bien à cette disposition par ses formes et parce que la vitesse moyenne dans la partie cloisonnée pouvait être de 8 à 9 $\mathrm{cm} / \mathrm{s}$ pour le débit maximum; on était donc bien en dessous de la vitesse à partir de laquelle les dépôts auraient été emportés par le courant vers le haut, ce qui eut été néfaste pour l'efficacité (2).

Comme on le voit sur la figure 2 , le cloisonnement divise l'espace en 5 batteries de tubes inclinés allant de 4 à $13,40 \mathrm{~m}$ de long. Les cloisons sont distantes entre elles de $0,60 \mathrm{~m}$, sauf pour

(2) La Honille Blanche, No spécial S.H.F.: Deuxièmes Journées de l'Hydraulique, $25-29$ juin 1952, P. Garnar et J.P. Raynavo : «Evolution de la décantation dans le passage de l'écoulement laminaire à l'écoulement turbulent et phénomènes de charriage dans les dessableurs à espace cloisonné ». 


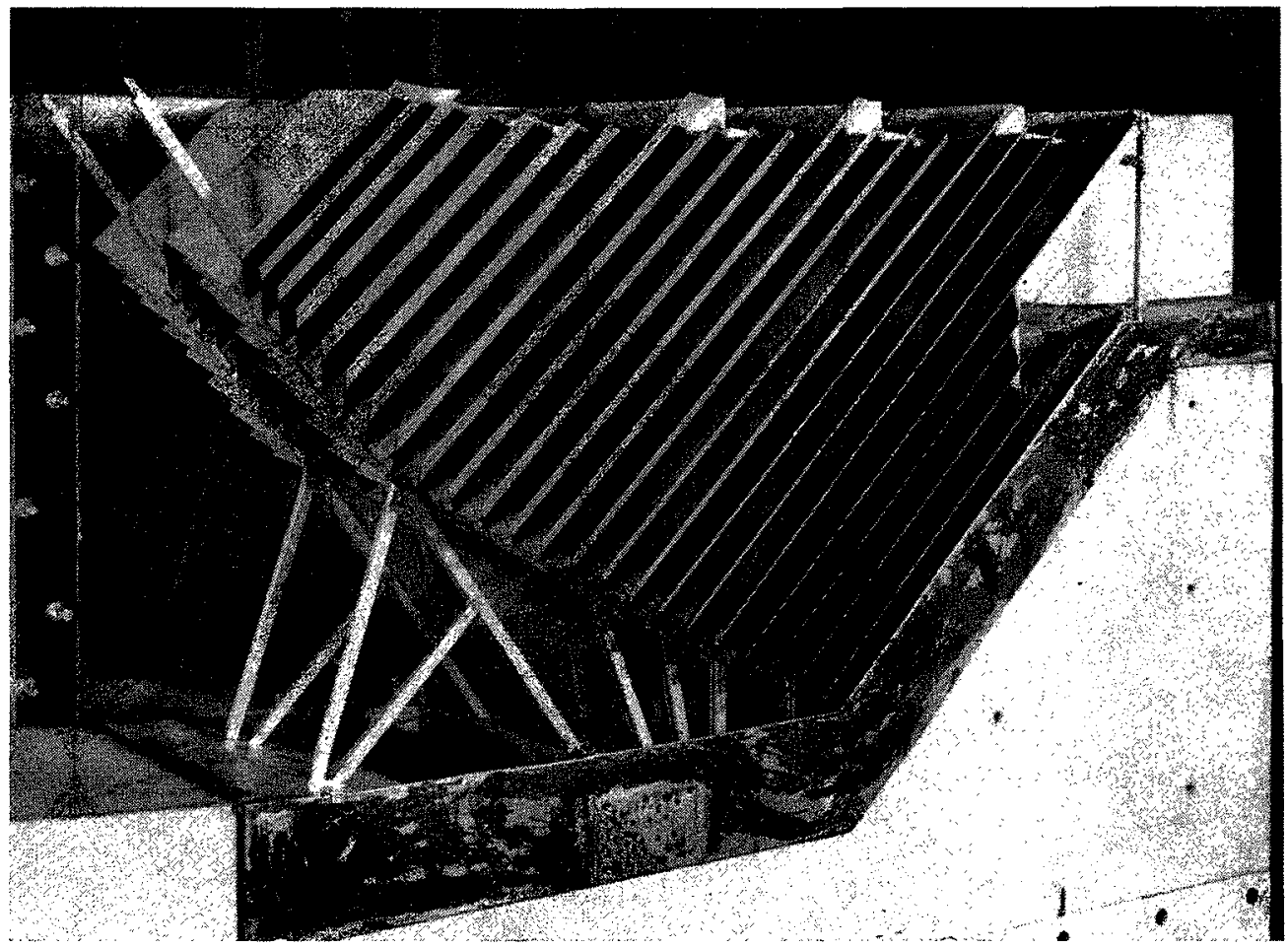

FIG. 4.

Cloisonnement essayé sur le modèle réduit.

les trois lubes les plus courts, pour lesquels cette distance a ćté ramenée à $0,45 \mathrm{~nm}$. La hauteur de chule libre des grains se trouve donc réduite à $0,85 \mathrm{~m}$ au maximum; elle est done dix à douze fois plus faible que la profondeur totale de l'ouvrage.

L'eau purifiée est évacuée par 5 fentes ménagées dans le plafond et par l'orifice aval. L'une des fentes se trouve en amont du cloisonnement et prend l'eau qui s'est décantée le long du plationd.

La figure 4 représente le cloisonnement essayé sur un modèle réduit au $1 / 20$, construit en plexiglass, ce qui permettait de bien roir les écoulements.

I.e but de ce modèle était :

- D'observer les écoulements el de vérifier le bon fonctionnement des organes de recueil;

- De déterminer les formes et dimensions des fenles d'évacuation de l'eau décantée;

-- De melle au point les dispositions permet. tant d'opérer facilement la purce permanente des sables fins sous le cloisonnement ef la chasse périodique des dépôts plus grossiers rassemblés sur le radier amont.

11 a permis également de faire cerlaines mesures d'efficacité pour comparer entre elles diverses solutions, mais non pour déterminer l'efficacité réelle de l'ouvrage, qui pouvait être calculée.
On a cherché sur le modèle les formes donnant la meilleure efficacití, en étant sûr que ces mêmes formes donneraient également la meilreure elficacité dans la réalité. Des disposilions particuliere ont permis de passer sur le modele un débit allant juscru'à 8 litres par seconde, alors que le débit maxinum en sinilitude de Froude était de $3,77 \mathrm{l} / \mathrm{s}$, de facon à avoir un nombre de Reynolds plus élevé dans le cloisonncment.

Grâce au modèle, il a élé possible de remédier à quelques défauts : d'abord en infléchissant vers le has le plafond inmédiatement à l'entréc et en plaçant deux grilles en série à l'aval du coude, on a pu annuler les perturbations d'entrée et alimenter uniformément la partie rectiligne de la chambre de dessablage. Sans cela, l'efficacité clait mauvaise.

On a pu régler les vitesses lans les tubes à des valeurs sensiblement proporlionnelles à leurs Jongueurs, alors que, sans précautions, if y avait certains lubes dans lesquels l'eau coulait en sens inverse du sens prévu. De mêne, on a ajuslé la forme des organes de recueil pour que le sable y descende aisément à lous les rógimes. Le modele a permis de constater qu'avec les formes definitives, le sable arrivait régulierement dans la fosse aval avec seulement une légère remise en suspension à la base quand l'eau était très chargée, mais le nuage ainsi formé se décantail dans les tubes les plus bas. 
Un autre point extrêmement important, que l'on a regardé de très près, concerne l'évacuation des dépôts. En effet, un dessableur ne peut pas fonctionner avec sécurité sans une évacuation irréprochable.

En marche normale, donc avec l'ouvrage en charge, les gros sables se déposent sur le radier amont et $\mathrm{y}$ restent immohiles, car les courants sont faibles; les sables fins se déposent sous le cloisonnement dans la fosse réservée à cel effet ef s'y accumulent beaucoup plus rapidement que sur le radier à cause du cloisonnement, si bien qu'il faut purger en permanence, sinon tout serait rapidement engorgé.

Une complication venait du fait qu'une seule vanne de chasse était prévue; cette difficulté a pu être tournée facilement. On a placé trois conduites aboutissant à l'amont dans la fosse des sables fins et à l'aval contre la face de la vanne à glissement. De cette manière, en levant cette vanne de $5 \mathrm{~cm}$, on peut assurer une purge des sables fins en perdant $60 \mathrm{l} / \mathrm{s}$ seulement; en levant la vanne de $20 \mathrm{~cm}$, cette chasse se fait à plus de $1 \mathrm{~m}^{3} / \mathrm{s}$, et en levant la vanne en plein, en même lemps que celle de la retenue, on vide l'ouvrage; le violent courant emporte alors tous les dépôts du radier.

Le dispositif a été complélé par une tuyauterie prenant l'eau dans la retenue et l'injectant dans les trois conduites d'évacuation des sables fins à chaque vidange, ce qui supprime tous les risques d'obstruction de ces conduites, quoi qu'il arrive. Le modèle montra que cette disposition présentait un intérêt, car ces conduites pouvaient s'obstruer lors des chasses sur le radier, qui pouvaient apporter plusieurs mètres d'épaisseur de sable sur ces conduites si elles étaient effectuées trop violemment.

\section{III. - EXPLOITATION DE L'OUVRAGE}

Les deux premières années d'exploitation ont permis de constater l'excellente efficacité de l'ouvrage comme la facililé de son exploitation.

Dès après la mise en eau en 1955 , une campagne de mesure fut entreprise en profitant des eaux de fonte des neiges, légèrement chargées en sable. On opéra par prélèvements continus dans des bidons de 200 lities ou avec des cyclones.

Bien que ces mesures aient été rendues délicates par suite des fluctuations de teneur à l'entrée de l'ouvrage, elles ont permis de tracer la courbe d'efficacité de l'ouvrage dont les éléments figurent dans le tableau II :

\section{TABLEAU II}

\begin{tabular}{|lr|r|r|r|r|r|r|r|r|}
\hline Diamètre & en $\mathrm{mm}$ & 0,4 & 0,3 & 0,2 & 0,150 & 0,100 & 0,075 & 0,050 \\
\hline Décanté & $\%$ & 99 & 98 & 97,5 & 95 & 72 & 53 & 30 \\
\hline
\end{tabular}

Les gros grains non arrêtés étaient des paillettes à faible vitesse de chute. A partir de $2 / 10^{\mathrm{c}}$ de $\mathrm{mm}$, il $\mathrm{y}$ avait des grains à peu près sphériques non arrêtés provenant de la fente amont non intéressée par le cloisonnement.

On peut caractériser l'ourrage en disant qu'il arrête $72 \%$ des grains de $0,100 \mathrm{~mm}$ et sensiblement tous les $0,2 \mathrm{~mm}$, ce qui est conforme aux résultats du calcul de la décantation dans les tubes élémentaires inclinés. La méthode de calcul tenait compte de la turbulence.

En comparant les chifres des tableaux I et II, on constate que l'on divise par des nombres com- pris entre 1,36 et 10 les quantités de sable de 0,05 à $2 \mathrm{~mm}$ qui échappent au dessableur, c'està-dire qui iraient user les machines. Comme les chiffres du tableau I étaient optimistes, on peut estimer que l'on diviserait par des nombres qui seraient plutôt compris entre 2 et 20 la quantité de sable, de respectivement 0,050 et $0,2 \mathrm{~mm}$, allant aux turbines.

Pour chiflrer le gain exact procuré par le cloisonnement, il faudrait connaître la granulométrie moyenne exacte du sable entrant dans l'ouvrage. Cela n'a pas pu être fait mais des essais sur le modile, avec el sans cloisonnement, en prenant un sable supposé représentatif de celui du torrent de la Trift, ont montré que, pour produire le même effet de décantation, il fallait prendre le dessableur cloisonné ou bien 3 à 4 dessableurs sans le cloisonnement. Evidemment, la topographie des lieux, comme aussi l'économie du projet, ne permettaient pas d'adopter cette dernière solution.

L'exploitation de l'ouvrage s'est faite sans difficulté, bien que les vannes soient télécommandées depuis plus de $15 \mathrm{~km}$. Elle sera encore facilitée par la prochaine mise en service d'un télétransmetteur d'épaisseur de sable Sineyrco qui permettra d'économiser en période de basses eaux sur le $1 \%$ de débit perdu pour la purge permanente, car on ne pratiquera les purges que lorsque l'appareil indiquera qu'il y a des dépôts en quantité trop abondante.

Il faut dire que la retenue arrête déjà beau- 
coup de matériaux : la presque totalité du sable plus gros que $0,4 \mathrm{~mm}$, ce qui soulage d'autant le dessableur. Mais celui-ci aurait fort bien pu s'accommoder des matériaux plus grossiers, allant jusqu'aux gravillons de 3 ou $4 \mathrm{~mm}$ sans nuire au bon fonctionnement de la purge permanente, qui n'est intéressée que par les sables fins. Mais le contrôle de l'imporlance des dépôts et de lopportunité des chasses cût été plus difficile.

\section{CONCLUSION}

L'installation d'un cloisonnement approprié dans le dessableur de la Trift a permis d'en améliorer considérablement l'efficacité. II arrête en effet $72 \%$ des grains de sable de $0,1 \mathrm{~mm}$ au lieu de moins de $18 \%$ sans ce cloisonnement.

Plusieurs délails importants onl été mis au point sur un modèle réduit qui a permis de choisir les formes les plus favorables.

Le résultat est que, finalement, sans changer le cadre général de l'ouvrage, l'efficacité obte- nue gràce au cloisonnement est la même que celle que lon aurait obtenue en partageanl le débit entre 3 ou 4 dessableurs en parallèle. De plus, les dispositions adoptées permettenl d'évacuer une grande partie des dépôts au prix d'une consommation d'eau infime: moins de $1 \%$ du débit traité.

Il semble bien que seul l'emploi d'un cloisonnement de l'ouvrage permellail d'alleindre un lel résultat.

\section{DISCUSSION}

(Président : M. Maurice Ganme)

N. le Président mpelle que l'appareil installé in silu à la suite des essais rapportés par M. Paul Garies a permis une alimentation de l'usine dans de très bonnes conditions. Il demande si le débit de $1 \%$, donné comme suffisant pour assurer, en l'espèce, l'évacuation des dépôts, a été obtenu déjà dans d'autres installations de dessableurs.

M. Remenieras indique que ee débit est, en général, cinq fois plus grand.

1. Labarr précise que l'on peut deseendre jusqu'à des débits de $2 \%$ avec des dessableurs discontinus mis en service par une horloge automatique, el plus bas avee des dégraveurs-dessableurs à détecteurs, mis au point par M. Molbert à la R.E.H. Alpes-II d'E.D.F., et en cours de montage à l'heure actuelle sur diverses prises.

Sur la demande de M. Molbent, relative à l'éventualite d'un dégravage préalable, M. Paul Garier indique que les gros grains peuvent se déposer sur le grand radier amont. du dessableur sans gêner le cloisomnement et être évacués par un courant violent après vidange de l'ouvrage (chasse discontinue); parallèement, une purge continue éracue tout ce qui est arrêté par le cloisonnement luimême. Par ailleurs, des essais faits sur le modèle en inclinant le radier ont permis de constater que l'on pouvait alimenter le dessableur eloisonné avec radier incliné $445^{\circ}$, done supprimer toute la chambre amont et avoir la mème efficacité.

Répondant à de nouvelles questions de M. MoLbent et de M. Labave, M. Paul Garmel indique que l'étendue du bassin rersant est de l'ordre ae $50 \mathrm{~km}^{*}$ avec $90 \%$ de la surface occupée par le glacier, et celle de la retenue, de $800 \mathrm{~m}^{2}$ environ; celle-ci atténue les faibles irrégularités du régime et l'exploitation fait des purges systemaliques, en principe, tous les dimanches et après les pluies d'orage.

En ce qui concerne l'utilisation du dessableu" dans le cas d'une prise en dessous, envisagée par M. Molnent, M. Paul Garres precise qu'un tel dessableur, associé à un dégraveur, est en cours de réalisation en Argentine; le souci de ne pas perdre trop d'eau conduit à réduire l'orifice de la purge permanente, et à dégraver a l'amont du dessableur, afin d'éviter que et orifice ne se bouche. Le dégravement se fait au moyen d'une simple fosse installé juste à l'amont du dessableur et dans laquelle les graviers tombent de facon continue. Les sables sont donc purgés par un orifice différent de celui du dégravenr.

Le risque de fortes pertes d'eau au cours du dégravage est réduit par son intermittence : il faut, néanmoins, une surveillance qui est sans doute évitée avee les dégraveurs automatiques avec horloge.

Sur la demande de M. Ponsand, M. Paul Cariel indique que leffeacité n'est reduite par la concentration qu'ì partir de 50 grammes par litre.

M. OUesner eslime que he dessablage des caux du torrent ayant essentiellement pour objet d'éviter une usure prématurée des aubes des turbines de l'usine, of cette usure ayant été constalée avec des suspensions de 5 a 10 microns, il serait intéressant de connâtre, pour des ritesses d'injection et des concentrations déterminées, linfuence du calibre moyen des particules en suspension, d'abord avec des limites inférieures et supérieures assez voisines (suspensions à granulometrie uniforme), puis avec des limites de plus en plus différenciées.

La réduction de la concentration, pour intéressante, essentielle même qu'elle soit, n'est pas en l'espece le but. recherche, le eritérium unique de la réussite de l'opération.

La connaissanee de linfluence du calibte des suspensions permettrat une meilleure orientation des processus de dessablage, et une meilleure adaptation des dispositions des dessableurs aux effets que l'on an escompte on la circonstance. Elle n'est done pas dénue d'intérêt, bien an contraire.

M. Paul Ganme indique que de telles mesures de granulométrio ont été failes à Innerthireben, mais qu'elles 
ne se sont pas étendues sur un temps sulfisamment long pour permettre des comparaisons valables; de toute facon, il ne parait pas possible de réduire l'usure due aux grains très fins.

M. Bonsix signale que l'usure des turbines de luate chute est due essentiellement aux grains fins en suspension. Ce phénomène pent être attribué cn grande partie au fait que les grains plus gros n'atteignent qu'une vitesse sensiblement plus faible que celle des jets d'une Pelton; à titre d'exemple, des grains de $3 / 10^{\circ}$ de $\mathrm{mm}$ peuvent n'emmagasinè que $10 \%$ de l'énergie cinétique qu'ils auraient a la vitesse du jet.

Des experiences conduites en labomtoire avec des sables de $1 / 10^{\circ}$ de $\mathrm{mm}$ et un jet de $38 \mathrm{~m} / \mathrm{s}$ environ ont monire que, à quelques decimètres de lorifice, l'action abrasive de la mixture etait croissante en fonction de la distance à lorifice (malgre une dispersion croissante du jet), ce qui confirme l'hypothèse précédente.

M. le Président souligne l'importance de la remarque de M. Bonne et pense que son hypothese devatit donnel lieu à des vérifications serrées.

M. Warry estime que, si l'on compare le dispositif présenté par M. Ganié à un dessableur classique relativement poussé, tel que celui de la centrale de Passy, il y a un gain de près de $1 / 10^{\circ} \mathrm{mm}$ à l'avantage du dessableur dinnertimehen : $72 \%$ des grains de $1 / 10^{\circ}$ de mm sont élimines dans cel ourrage, alor's qu'à Passr, ce rendement n'est obtenu que pour les grains de 2/10es mm.

Par contre, si l'on compare le dessableur prévu à l'orígine à Innertilichen au dessableur de Passy, on constate que, pour un volume d'ouvrage par $\mathrm{m}^{3 / \mathrm{s}}$ traité légèrement inférieur, le dessableur de Passy semble avoir une efficacité sensiblement supéricure à celui d'Innertkirchen qui n'éliminait que 56 \% des grains de $2 / 10^{\mathrm{s}} \mathrm{mm}$.

Cette efficacité réduite semble liée à quelques imperfections non négligeables : grande profondeul, longueur assez l’éduite, conde à l'entrée. De plus, la présence d'un fond a faible pente, avec une seule série d'orifices do purge à l'aval, peut laisser craindre la formation de depôts importants sur ce fond, pouvant provoquer lobstruction des orifices par éboulement lors d"un incident. de marche, comme cela a été constaté sur certains onvrares des Alpes. Si, au contraire, on ne constate pas de dépôts importants dans cette partie de l'ouvrage, cela ne provient-il pas de ce que, dans le cas particulier, la retenue elle-meme forme décanteur pour les sables les plus grossiers?

En ce qui concerne la différence entre les taux d'épuration réels et les valeurs théoriques utilisécs par $M$. Ganiel, M. Matrue précise que les mesures effectuées, cn 1953 , à Passy par le Service de M. Bourguignow, montrent que, pour les grains de l'ordre de $2 / 10^{\mathrm{ss}} \mathrm{mm}$, la concot.. dance reste bonne pour les vitesses d'écoulement considérécs $(0,40 \mathrm{~m} / \mathrm{s}$ environ).

M. MaIre pense, d'autre part, qu'un problème imporfant est celui de la composition granulométrique des sa- bles lransportes par lo lorrent : des mesures eflectués ces dernières années dans des usines dont le bassin versant comporte 20 a $25 \%$ de glaciers (Passy et Pont Escot. fier) ont montré que les sables en suspension a l'entréc de ces prises d'ear comportaient $80 \%$ d'éléments inférienrs à $1 / 10^{\circ} \mathrm{mm}$ et environ $50 \%$ d'éléments inférieurs à $6 / 100^{\mathrm{es}} \mathrm{mm}$. Il serait intéressant de connaître la granulométrie des grains contenus en suspension a l'entré du dessableur d'Innertkirchen, car on peut se demander si, dans un bassin versant glaciaire dans sa quasi-totalité, la proportion d'éléments très fins échappant même à laction du dessableur cloisonné ne risque pas d'ctre plus clevée. Ce point est important daus la mesure ou il conditionne, avee le prix de revient du cloisonnement, la rentabilité économique de l'ouvrage.

M. Paul Ganner répond qu'en fait, si les conduites de purge n'ont pas éte bouchées, cela ne veut pas dire qu'il n'y ait pas eu des couches épaisses de sable sur le radier; malheureusement, le détecteur mécanique ne fonctionnail pas à l'époque à laquelle ont été faits les essais et il sera, d'ailleurs, remplacé par un autre appareil, qui transmettra électroniquement lépaisseur du sable dans la fosse. Il $y^{x}$ a eu des dépôts considézables, hien que la retenue ait une surface assez grande : on a vu des quantités énormes de sable s'en aller au cours des chasses périodiques.

D'antre part, il est bien cxact que Ia grande majorité des grains sont trè's fins et de ce fait, il semble qu'on alteigne par ce procédé du cloisonnement la limite économique des dessableurs : on ne gagnerait pas beaucoup en diminuant les vitesses on par une augmentation du cloisonnage. La recherche d'une plus grande efficacité théorique, en utilisant un tonnage de métal égal à celui du cloisonnement, conduiraì à l'emploi d'autres systèmes, tcis que des cyclones; mais ces appareils ne fonctionnent convenablement que s'ils sont de petites dimensions, done nécessairement associés à des grilles à nettoyage automatique, ce qui conduit à un tonnage d'appareils finalement plus important, à une complication mécanique et à une perte de charge généralement peu admissible.

M. IABAye pense que, lorsque les dépenses dues au des sablage croissent trop, le compromis le plus économigue consiste, sans doute, à traiter spécialement les aciers des turbines pour leur permettre de résister aux matériaux transportés, ou bien à remplacer les turbines de temps en tamps.

M. Paul Garier estime que ce compromis est à rechercher dans chaque cas de qu'en ce qui concerne Innertkixchen, l'examen économique a montré qu'il fallait en promier lien accroitre l'effeacité du dessableur initialement prévu.

Sur la demande de M. le Président, M. Paul Garter. indique qu'un autre dessableur du même genre est en construction an Pérou : il est a éconlement horizontal et traitera, dans le futur, $48 \mathrm{~m}^{3}$ a la seconde.

M. le Président remercie le confërencier. 